39

\title{
A novel method to create intelligent sensors with learning capabilities to improve modern production systems
}

\author{
A. Mahajan \\ Associate Professor \\ School of engineering and Mathematics \\ Lake Superior State University, Sault Ste. Marie, Aff 49 783, \\ USA \\ Tel: $\quad 906-635-2598$ \\ Fax: 906-635-6663 \\ Email:amahajan@lakers.Issu.edu
}

\begin{abstract}
A formal theory for the development of a generic model of an autonomous sensor is proposed and implemented. An autonomous sensor is defined as an intelligent sensor that has machine learning capabilities. It not only interprets the acquired data in accordance with an embedded expert system knowledge base, but is also capable of using this data to modify and enhance this knowledge base. Hence, the system is capable of learning and thereby improving its performance over time. The main objective of the model is to combine the capabilities of the physical sensor and an expert operator monitoring the sensor in real-time. The system has been successfully tested using various simulated data sets as well as a real thermistor that has been developed as an autonomous sensor. This work has significant impact on modem production systems since sensors form an integral part of all closed loop control systems, and modem manufacturing processes rely heavily on sensor based control systems. The long range aim of this work is to develop highly autonomous production systems that have self diagnostic, maintenance, self correction, and learning capabilities embedded at the local and global levels. This work builds upon work on a formalized theory for autonomous
\end{abstract}


sensing called Dynamic Across Time Autonomous - Sensing, Interpretation, Model learning and Maintenance Theory (DATA-SEIALAMT) that has been supported by the NSF and the SME Education Foundation.

\section{Keywords}

Intelligent sensors, learning, autonomous, modem production systems

\section{INTRODUCTION}

Sensing has become increasingly important in the control of complex and sophisticated systems of today's technology. There is an increased interest in developing a general theory to treat intelligent sensor systems [1,2,3]. Parallel work has been done in industry where sensors have been developed with built in expert systems and look-up tables [4,5]. These sensors, called smart sensors, are described as simple sensing devices with built-in intelligence. This intelligence includes decision making capabilities, data processing, conflict resolution, communications, or distribution of information. Again, there is no generic model, and the sensors and their built-in-intelligence usually very specific towards their field of application.

The autonomous sensor is defined as a sensor that has an expert system with extensive qualitative tools that allow it to evolve with time into a better and more efficient system [6]. It differs from the above mentioned models by having a dynamic knowledge base as well as embedded qualitative and analytical functions that give it a higher degree of operational independence, self-sufficiency and robustness. The underlying philosophy behind the autonomous sensor is probably closest to Henderson's [7,8] logical sensor models that also endeavor to give more problem solving capabilities to the sensor.

Multisensor integration and fusion has always been an attractive method to reduce the ambiguity in the sensed data, and considerable work has been done in this area $[9,10,11,12]$. The autonomous sensor model can reduce the need for redundant sensors, and give more accurate information for integration and fusion purposes. Recent work in qualitative reasoning regarding physical systems has been published in a special volume of Artificial Intelligence [13]. This volume focuses on the development and use of qualitative reasoning methods to cope with fundamental engineering tasks. A link has been forged between qualitative reasoning and the traditional numeric and analytic methods.

DeCoste [14] describes a system, called DATMI, that dynamically maintains a concise representation of the space of local and global interpretations across time that are consistent with the observations. Each of the observations is obtained from a sensor, and therefore the number of observations is equal to the number of sensors in the control system. The truth of the observations and the validity of the 
sensors is obtained by cross-referencing with possible and impossible states of the system (envisionments). DATMI is designed for a complete control system comprising of multiple sensors and actuators, and is the inspiration for the formalized theory developed by the authors [15]. This theory is called DATASEl4LAMT (Dynamic Across Time Autonomous Sensing, Interpretation, Model Learning and Maintenance Theory) and is designed for and is applicable to each sensor in the control system.

Brooks $[16,17]$ states that a truly intelligent system would perform its own abstraction of the problem and then solve it. He has also presented the subsumption architecture and used it successfully on various mobile robots developed at the MIT Laboratory of Artificial Intelligence. The central theme is that there is no central representation or an overall intelligent controller. This entire philosophy of perception and reaction without a specific intelligent controller is also in keeping with the philosophy underlying the autonomous sensor that allows more decision making and problem solving at the sensor level rather than at the controller level in a robotic, or other control system.

The autonomous sensor model is really a combination of a sensor and an operator monitoring it in real time. The operator has a basic understanding of the sensor and the measurand (entity being measured), and as time passes he learns more about the sensor and measurand behaviors and uses his knowledge to keep running the sensor in as perfect a condition as possible. It is the operator's part that has to be embedded in existing sensors to make them autonomous.

The autonomous sensor finds applications in any physical system that uses sensors since the sensors themselves become more robust and reliable. It is particularly suited for applications where the installed sensors can not be accessed for long periods of time, e.g. nuclear power plants, space structures, etc. In such applications the autonomous sensor could function for long periods of time fairly well without going through hard calibrations at short intervals of time. It would also be able to better handle itself in adverse conditions. Autonomous sensors are also useful in situations where the system has a large number of sensors, since installation, calibration and monitoring done by the sensor itself can greatly decrease the complexity of the system's operation. Examples are automated systems in industry, ships, airplanes, shuttles, etc.

\section{DATA-SIMLAMT (Formalized Theory)}

DATA-SAMT is a philosophy that has been inspired by the need for autonomous sensors, and these in turn were inspired by the need for autonomous systems. DATA-SEQLAMT, as presented in this paper, is a formalized theory to create autonomous agents, and the autonomous sensor is an application of this theory. 


\subsection{The Different Types of Databases}

DATA-SIMLAMT defines two types of databases for the autonomous sensor. These databases keep a running history of the sensed value in the quantitative and qualitative domain.

a) Quantitative Database: This consists of a history of the actual numerical values sensed by the sensing element, and some pertinent statistical parameters and their numerical values, e.g. mean, std. dev., etc.

b) Qualitative Database: This database consists of qualitative symbols that represent some situation of a relevant aspect of the system at that particular instant. These symbols are defined based on limiting quantitative parameters. These relevant aspects are called properties, and the symbolic representations are called state values.

Some of the terms that will be used in this paper are defined as follows:

Property - is a parameter, e.g. amplitude check.

Concept - is a set of same properties, e.g. amplitude is high for a duration of time.

Behavior - is a set of concepts, e.g. a normal operation followed by a very high amplitude may be a spike.

Envisionment - is a known, hence pre-defined, concept or behavior similar to a known pattern in the pattern recognition problem.

\subsection{Windowing}

DATA-SIMLAMT uses three types of windows to determine the number of samples that must be used to define the properties, concepts and behaviors. These windows operate on the various databases described in the previous sections. Windowing is an important feature of DATASIMLAMT, and provides observation histories of appropriate length to identify the different

behaviors in the sensor and measurand domain. These are dynamic windows, i.e., they can change size under various circumstances.

Measurand Identification Window: This window is used to obtain the instantaneous model of the behavior of the measurand. This is essential for the sensor to identify problems in its own operation. DATA-SE\4LAMT attempts to give the sensor with various analytical modeling and tracking routines so that it can use the one that best captures the instantaneous behavior of the measurand.

Quantitative Database Window: The main function of this window is to capture transitions. This window operates on the raw data as is sensed by the physical sensing device, and carries out pre-defined statistical and identification functions so as to supply enough information for the creation of the qualitative database. This window sets the number of points to be used in functions such as standard deviations, moving averages, etc. 
Qualitative Database Window (QLW): This window acts upon the qualitative data base which has been created based upon the properties and their state values. This is the most important window as it matches the real-time qualitative data to the envisionments in the knowledge bases to come up with interpretations and identify sensor and measurand behaviors. Essentially, each behavior has a window which is dictated by the combination and sequence of its associated concepts.

\subsection{DATA-SIMLAMT knowledge bases}

DATA-SFMLAMT has two types of knowledge bases that are described below.

Internal knowledge base: This consists of the basic knowledge that is required to distinguish between the normal behavior of the sensor and any other behavior that differs from the pre-defined normal behavior. This knowledge base also has knowledge such as the time response of the sensor, the time required for a change in the measurand, appropriate methodologies for changing the size of the windows, analytical tools such as statistical routines, fast fourier transform routines, curve fitting routines, least-mean-square adaptive algorithm, etc. The internal knowledge base is a generic part of the model that can only be changed at the software level, i.e., it requires some programming effort on the source code.

External knowledge base: This knowledge base is the key feature of DATASIMLAMT, and hence, of the autonomous sensor. This knowledge base resides in external data files that may be created by the operator, and includes the specification sheet of the sensor and files containing the concepts and behaviors. These may be modified and updated by the operator as new behaviors are discovered, or the process may be automated, i.e., the knowledge base may be updated by the sensor as it discovers new behaviors that do not match the normal behavior of the sensor. The sensor memorizes new behaviors, identifies appropriate countermeasures for them, and categorizes them such that it may use them the next time they appear. This constitutes the learning mechanism of DATA-SIMLAMT, and will be discussed in a later section.

\subsection{The Generic Model for an Autonomous sensor}

This section gives the operational model of an autonomous sensor. The model has sensing, interpretation, truth maintenance and model learning capabilities embedded in it as can be seen in Figure 1. Data travels from the physical sensing element to the interpretation module, and then the maintenance and learning modules. These modules obtain knowledge to act upon the sensor data from the various knowledge bases in the sensor as well as the measurand domains. 


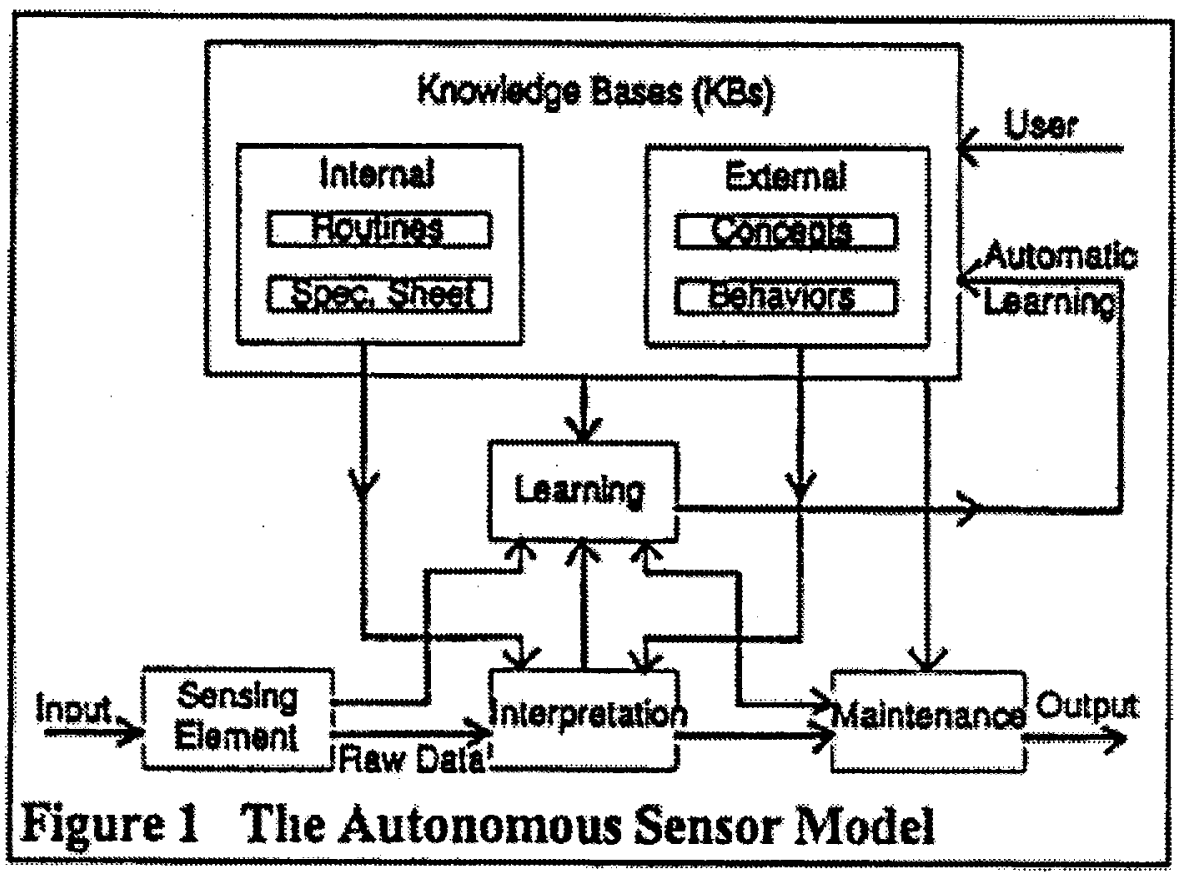

\section{ENVISIONINIENTS (or Patterns)}

The envisionments are the most important feature of DATA-SIMLAMT, and were introduced before as part of the external knowledge base. These envisionments describe measurand behaviors such as step changes, etc., and abnormal sensor problems such as spikes, disturbances, dead sensors, etc. The sensor must distinguish between the behaviors that encompass the two different domains (measurand and sensor), since the sensor needs to take appropriate countermeasures if there is a sensor problem, but simply follow faithfully a valid change in the measurand behavior. These envisionments reside in external ASCII data files and can be created by the operator or by the sensor itself with the help of specialized machine learning algorithms. The sensor can use these precompiled envisionments, or it can start with a blank external knowledge base and create the very same envisionments during its operation when the learning mechanism is switched on.

Let us investigate a sudden jump in the signal from a normal value to a very high value. When there is a sudden jump in the signal it could have been caused by one of the following three occurrences:

a) It could be caused due to a problem in the internal circuitry of the sensor due to a surge of current, or a short circuit. It must be noted here that there are 
sometimes two distinct circuits, one associated with the sensor, and the other associated with the data-acquisition system. For simplicity, the effect of both will be grouped together under sensor behaviors.

b) It could be a start of a valid step change in the measurand.

c) It could be due to an external disturbance.

How does one quantify the term sudden? The sensor and the measurand time constants should always be checked against the actual time taken for the measurand to change from one value to another. A step change is the "most sudden" possible change in the measurand behavior. Hence, the following four possibilities can arise out of the comparison of the actual time (AT) with sensor time constant (STC) and the measurand time constant (MTC). Remember, the sensor time constant is always less than the measurand time constant (STC < MTC).

i) If the actual time elapsed during a change in the sensed signal is faster than the sensor time constant (AT < STC), then it has definitely been caused by a problem in the internal circuitry of the sensor due to a problem such as a surge of current, or a short circuit. This behavior can confidently be interpreted as a Spike. If this continues for more than one or two samples then the behavior is an internal disturbance due to a sensor problem, and will be denoted by the following behavior and state value Disturbance(Sensor).

ii) If the time elapsed during a change in the sensed signal is greater than the sensor time constant but less than the measurand time constant (STC $<$ AT $<$ MTC), then it is definitely an external disturbance due to foreign body. This is because the sensor is responding to a valid change in a measurand behavior and hence does not violate the sensor time constant condition, but does violate the measurand time constant condition. This will be denoted by the following behavior and state value - Disturbance(Measurand).

iii) If the time elapsed during a change in the sensed signal is equal to the measurand time constant (AT $=\mathrm{MTC}$ ), then it can be presumed that the measurand has gone through a valid Step Change, and the sensor is operating normally.

iv) If the time elapsed during a change in the sensed signal is greater than the measurand time constant (AT > MTC), then it can be assumed that the sensor is operating normally and is following the measurand behavior. It could also be disturbance from a foreign body whose time constant is greater than the measurand, but there is little one can do in this case except follow the change. 


\section{4, DATA-SIMLAMT AT WORK}

There are some basic assumptions that must be made for the smooth and valid operation of the autonomous sensor, and these are:

a) The measurand behavior must be continuous in time, and if not, should be modeled as such. A thermistor measuring the room temperature continuously for some control system can easily be instantiated as an autonomous sensor. But, if this thermistor is used to measure the temperature for intermittent periods of time then the time intervals when the thermistor is not being used should be treated as zero-time gaps.

b) Since the measurand is itself usually a controlled variable it has its own response time to a step change. The sensor time constant, defined as the time for it to change 63.2 percent of the total difference between its initial and final value when subjected to a step change, should be less than the measurand time constant.

c) The sampling time of the data acquisition system should be less than the sensor time constant.

This is essential in capturing transitions in the measurand behavior, and is a necessary design criterion.

d) The sensor should have some idea about the frequency content of a harmonic signal. This is essential to extract and identify the principle frequencies of the signal and to be able to isolate it from a possible high frequency noise in the signal. In a non-harmonic signal this is not necessary, since the high frequency components can be assumed to be the noise contents.

DATA-SIMLAMT has four main tasks associated with it, these being sensing, interpretation, maintenance and learning (Figure 1 shows the different modules as well as the flow of information). The autonomous sensor model will be demonstrated using a specific sensor, i.e., a thermistor. This thermistor may be part of a control system that needs to know the room temperature for control purposes, and is using the thermistor to measure it.

Sensing includes two stages based on the traditional model of a sensor that consists of a physical sensing device that senses the measurand in some manner, and an analytical model that converts the sensed quantity into a digital value representative of the measurand.

Interpretation is the process which includes the creation of the quantitative database, then the qualitative database, and then the final matching of the realtime qualitative database with the envisionments to come up with the final interpreted behaviors in the sensor and measurand domain. This is similar to the pattern recognition problem as mentioned earlier. The matching is done by incrementing a Matching Function (MF) every time a property and its state value 
is matched. A perfect match means that $\mathrm{MF}=100 \%$. A behavior can only be interpreted if the associated $W$ has a value of 100 . This process can also be termed as the diagnostic component of DATA-SEI4LAMT. This is the process very similar to a typical pattern recognition process.

Maintenance is the process of correcting the sensed value in some manner if a sensor problem has been interpreted, filling gaps in the quantitative database, and resolving conflicts by consulting possible and impossible states of the system and the recent history of the sensor and measurand behavior. The main hindrance to the autonomous sensor is that it does not have a model of the measurand behavior so it infers a reliable instantaneous model of the measurand behavior at all times, and only then can it identify its own problems as they occur. This instantaneous model is obtained by using various modeling tools available to the autonomous sensor, and is done within the Measurand Identification Window. The various analytical tools available to the autonomous sensor are FFT analysis routines, FIR filter design routines, curve fitting routines, LMS adaptive predicting routines, etc. The maintenance process uses knowledge from the, envisionments and the specification sheet that includes the quantitative parameters, tables for drift, and other data.

Machine learning is an important capability for any autonomous system, but one needs to know what constitutes learning before one can call it learning. The following definition of learning is proposed by the authors:

Learning, as applied to physical systems, may be defined as a process in which the system modifies its existing model (the structure itse6C or simply the parameters of the existing structure) based on the information extracted from the input data according to pre-defined guidelines or principles (performance measure/critic), memorizes the change, and has the ability to use this acquired knowledge in the future. The system should be able to adapt in some manner while it is operating even when there is no human involved in the closed loop system.

The autonomous sensor has two levels of learning. The first is the learning of quantitative parameters, and the second is the learning of qualitative behaviors that comprise the external knowledge base.

Learning of Quantitative parameters: This is a lower level of learning in which the sensor parameters are fine tuned against an assumed measurand behavior. This is similar to system identification, except that the sensor does not have an exact model of the input, but has an assumed instantaneous model of the measurand behavior. Usually, the analytical model of the sensor should only be changed during a hard calibration done against known input signals. However, there are cases where some of the sensor constants can be changed with a fair 
degree of confidence. For example, whenever the sensor is certain that a step change has occurred, then the currently recorded measurand response time can be used in the future rather than the initially recorded one.

Learning of Qualitative behaviors: This is a higher level of learning in which the sensor can recognize new behaviors that differ from the normal behavior, classify them according to predefined principles and guidelines, memorize them, and use this acquired knowledge in the future. The aim, of course, is that the sensor continuously improves with time. This is manifested in a reduced amount of time spent on interpreting previously learned behaviors, and in an improved performance in countering problems.

The autonomous sensor model is based on the philosophy of making the sensor self-sufficient One can make the sensor self-sufficient by defining a perfect model for it under all circumstances, incorporating all the possible problems that it could encounter in its operation, and all the possible measurand models that it could encounter. But, this assumes the fact that the knowledge about sensor and the measurand is absolutely complete and can be coded in without mistakes and missing pieces. If for any reason this assumption does not hold, then one has to assume that the sensor is not self-sufficient, and needs expert human support.

The envisionment of the normal behavior of the sensor is used as a critic, called the Normal Behavior Segment. All real-time segments in the qualitative data base are compared to this envisionment, and if the matching function (MF) has a value of $100 \%$, then the segment is said to have passed the Normal Behavior Interpretation Test. This algorithm to distinguish between a valid change in the measurand behavior and an abnormal sensor behavior is based on the assumption that if there is a sensor problem then there has to be a recovery some time in the future.

Formalizing a methodology to distinguish between a sensor problem caused due to the internal circuitry and one caused due to the actual physical sensing element is a difficult task. DATASIMLAMT provides an initial attempt to do so by watching trends in the quantitative corrected raw data base to capture a possible problem in the physical sensing device. This problem occurs over a long period of time and usually manifests itself as a drift in the sensed values. It is usually compounded by high temperature violations and repeated harmonic cycles. If pre-compiled tables for drift are not available then the sensor can, under certain circumstances, compile its own tables.

Since drift is compounded by harmonic cycles, DATA-SEMLAMT keeps track of the harmonic cycles and an average value obtained by recording successive local maximas and minimas, and then finding a correlation between them. This high level approach is only valid for a periodic signal. The advantage of this algorithm is that the signal need not be a perfect sinusoid, but can be any periodic signal with various frequencies and noise components. 


\section{SIMULATIONS AND EXPERIMENTS}

This section presents the performance results of the autonomous sensor implementation. The following three figures show the raw data (dotted lines) and the corrected data (solid lines).

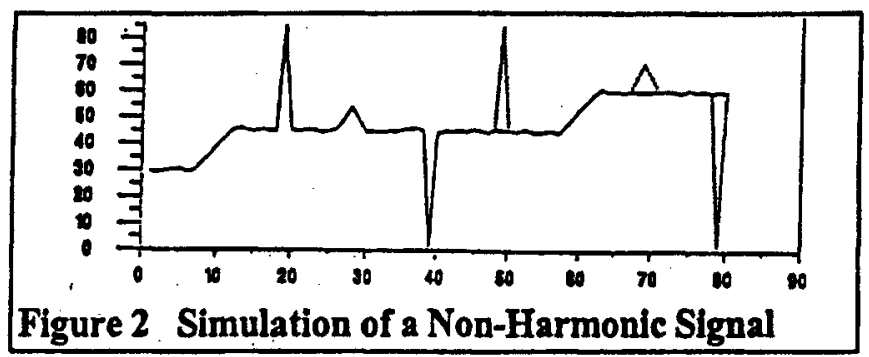

Figure 2 shows the raw signal with numerous faulty data points due to problems such as spikes, stc. Notice, the sensor is able to follow a step change, and leams about spikes and dead sensors the arst time, and uses the knowledge later on to correct the original data.

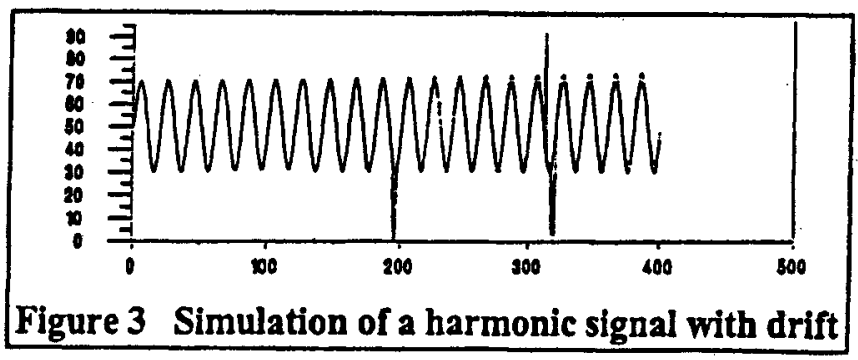

Figure 3 shows a harmonic signal that has drifted with time, and has spikes. The sensor recognizes the spikes and the drift, and compensates for the faulty raw data. 


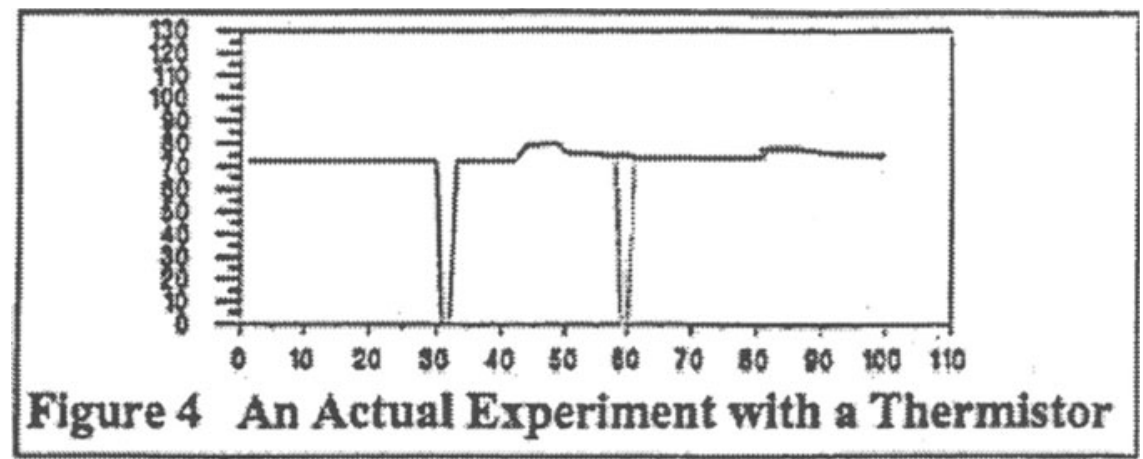

Figure 4 shows the results of an experiment in which the autonomous sensor model was used in conjunction with an actual thermistor measuring room temperature. The sensor was deliberately switched off twice and the temperature changed. Notice, the sensor follows the first problem, but then learns about it and compensates for the problem the second time around. It also recognizes the changes in the room temperature, and follows them faithfully.

\section{CONCLUSIONS AND RECOMMENDATIONS}

A formal theory for the development of a generic model for an autonomous sensor has been proposed and implemented. This theory, called DATASIMLAMT, attempts to formalize knowledge that had formerly remained in the domain of the human operator. The autonomous sensor model is an advanced version of the existing smart or expert sensors that only include an embedded expert system. The improvement is in the form of learning capabilities that allow the model to evolve with time for improved performance. Future work entails increasing the number of analytical tools 'lable to the sensor in modeling the measurand behavior as well as using optimization techniques for the interpretation process. The autonomous sensor model will be used to improve the accuracy of an ultrasonic 3D position sensing system being currently developed at LSSU. 


\section{ACKNOWLEDGMENTS}

The authors wish to thank the National Science Foundation (Grant \#HU-9119178) and the Society of Manufacturing Engineers Education Foundation (SME 596-233 1) for supporting this work.

\section{REFERENCES}

1. Ghani, N., "Sensor integration in ESPRIT," IFAC Proceedings, Karlsruhe, FDR 1988, pp. 323-328.

2. Pinkava, J., "Towards a theory of sensory robotics," Robotica, Vol. 8, 1989, pp. 245-256.

3. Lozano-Perez, T., Mason, M. T., and Taylor, R., "Automatic synthesis of fine motion strategies for robots," Int. J ofrobotics Research, Vol. 3, No. 1, 1984, pp. 2-24.

4. AbdelRahman, M. and Smith M. L., "The Impact of AI On Sensing Technology," SENSORS, September 199 1, pp. 16-22.

5. Studt, T., "Smart Sensors Widen Views on Measuring Data," $R \& D$ Magazine, March 1994, pp. 18-20.

6. Figueroa, F. and Mahajan, A., "Generic Model of an Autonomous Sensor," Mechatronics, Vol. 4, No. 3, pp. 295-315, 1994.

7. Henderson, T and Shilcrat, E., "Logical Sensor Systems," Journal of Robotic Systems, 1(2), 1984, pp. 169-193.

8. Henderson, T., Hansen, C. and Bhanu, B., "The Specification of Distributed Sensing and Control," Journal ofrobotic Systems, 2(4),1985, pp. 387-396.

9. Dur-rant-Whyte, H. F., "Sensor Models and Multisensor Integration," International Journal of Robotics Research, Vol. 7, No. 6, 1988, pp. 97-113.

10. Luo, R. C. and Kay, M. G., "Multisensor integration and Fusion in Intelligent Sytems," IEEE Transactions on Systems, Man, and Cybernetics, Vol. 19, No. 5, September, 1989, pp. 901-931.

11. Kuc, R. and Siegel, M. W., "Physically Based Simulation Model for Acoustic Sensor Robot Navigation," IEEE Transactions on Pattern Analysis and Machine Intelligence, Vol. PAMI-9, No. 6, November 1987, pp. 766778 .

12. Elfes, E., "Sonar-Based Real-World Mapping and Navigation," IEEE Journal of Robotics and Automation, Vol. RA-3, No. 3, June 1987, pp. 249265.

13. Guest Editors: de Kleer, J. and Williams, B. C., "Qualitative Reasoning about Physical Systems," Artificial Intelligence, Vol. 5 1, No. 1-3, October 1991.

14. DeCoste, D., "Dynamic Across-Time Measurement and Interpretation," Artificial Intelligence, Vol. 51, 1991, pp. 273-341. 
15. Mahajan, A. and Figueroa, F., "Dynamic Across Time Autonomous Sensing, Interpretation, Model learning and Maintenance theory (DATASIMLAMT)," Mechatronies, Vol. 5, No. 6, 1995, pp. 665-693.

16. Brooks, R. A., "Intelligence without representation," Artificial Intelligence, Vol. 47, 199 1, pp. 139-159.

17. Brooks, R. A., "Robot Insect - Attila," Popular Science, March 199 1, pp.52-55, 86 . 